\title{
User Experience Research in the Semiconductor Factory: A Contradiction?
}

\author{
Marianna Obrist, Wolfgang Reitberger, \\ Daniela Wurhofer, Florian Förster, and Manfred Tscheligi \\ Christian Doppler Laboratory for "Contextual Interfaces", \\ HCI \& Usability Unit, ICT\&S Center, University of Salzburg, \\ Sigmund-Haffner-Gasse 18, 5020 Salzburg, Austria \\ firstname. lastnamedsbg.ac.at
}

\begin{abstract}
No doubt, user experience (UX) has become of high relevance within the HCI community. Within this paper, we present initial results from a qualitative study on UX in the factory context, more precisely in a semiconductor factory. We highlight the challenges of performing UX research in this particular context and the usefulness of probes for collecting feedback from operators in the factory context within in a limited timespan. The results provide an initial rich description of the operator's everyday experiences in a semiconductor factory. From a designer's point of view, this allows for a more empathic interaction design informed by a subjective operator perspective.
\end{abstract}

Keywords: user experience, factory context, probing, user study.

\section{Introduction and Motivation}

So far, only little work focusing on the interaction and user experience (UX) in factories has been done. However, UX in a factory context is crucial. For example, cooperation and reliability of workers - often mediated through technology - are essential for the course of actions in the factory. Human errors resulting from poorly designed interfaces lead to high costs and even defective end products. Additionally, emotions - although not primarily associated with factory work - might influence the cooperation between factory workers as well as efficiency and reliability. Thus, UX and the related methodological challenges in the factory context represent an important research topic. Within this paper we introduce how we dealt with the challenges and limitations in a semiconductor factory when conducting an experience-focused user study. We will show how we successfully adopted the probing method for investigating UX in this particular context.

First of all, to create awareness and foster appreciation of UX research in the factory, a workshop with members of the factory management was held. The research goals and the benefits of a qualitative study were discussed. Initial doubts on whether a creative method like probing would work for a group of fairly unskilled workers, were dispelled. At the end, the method proved to not only work well but also lead to relevant findings. The adapted probing method engaged the operators (i.e. workers) and motivated them to give a rich account of various aspects of their everyday work 
experience, including open input about perceived negative issues such as stress. The deeper understanding of the subjective perception that operators have of their work context has the potential to better inform designers and increase their empathy for this particular user group.

\section{UX Research in a Semiconductor Factory Context}

In the following we describe the potential of UX research for the factory context considering the particularities of a semiconductor manufacturing plant and the resulting methodological challenges.

\subsection{Relevance of User Experience Research}

Following Hassenzahl and Tractinsky [7], UX is a consequence of a user's internal state (e.g., predispositions, expectations, needs, motivation), the characteristics of the system (e.g., complexity, usability, functionality, etc.) and the context where the interaction occurs (e.g., organisational or social setting). UX itself can be understood as a dynamic, complex and very subjective phenomenon [10]. Moreover, according to Alben [1], UX comprises all aspects of how people use an interactive product: the way it feels in their hands, how well they understand how it works, how they feel about it while they are using it, how well it serves their purposes, and how well it fits into the entire context in which they are using it. This definition particularly points out the relevance of UX research in all areas where humans interact with a system, thus also in a semiconductor factory context, where people are increasingly confronted with new technologies and user interfaces to complete their tasks.

\subsection{Understanding the Context and Challenges}

The main challenge for semiconductor manufacturing plants is the coordination of many operators working on different machines to guarantee an efficient and error free production process. As soon as we talk about factory ergonomics like usable, safe, and comfortable interfaces, we are also addressing aspects of UX. Despite the obvious relevance of UX in the factory, little research has dealt with this issue. This could be partly rooted in the difficulties and limitations such a context involves (see below) or due to the fact that the investigation of UX might lead to competitive advantages, and are thus not published for a greater audience.

One particular challenge of this context is the "clean room" with its special environmental conditions. In a clean room it is necessary to wear a special outfit covering the whole body and to use a mask. Traditional study materials, such as paper and pen, recorders, etc. are not allowed to take into the clean room. Additionally, shift-production circles - the manufacturing plant operates 24/7 with day and night working shifts - can also impede a field study in this context. Consequently, doing a field study on UX in a semiconductor context often requires modifications and adaptations of traditional methods.

For decades scientists were and are still being occupied to investigate factory work from a point of view of classical or social psychology [3]. The factory context is a wide area of research within the field of ergonomics, also focusing on technologies 
and its impacts on workers, especially occupational safety and health issues [17]. However, little research has been done to investigate UX issues within a factory context so far (e.g. [8]). Few materials can be found that put factory workers into the focal point of attention when designing interfaces. In the beginning of the 90s an "IEE Colloquium on HCI: Issues for the Factory" was held in London, dealing with psychological basis for computer system design, operator support systems and industrial inspection [13], involving an article on the design of operator-computer interfaces that give operators an accurate picture - acquisition and display of data and allow the control of the production process. Going a step further and trying to understand how operators feel about and experience the interaction with diverse interfaces, is still rare with respect to the increasing relevance of UX research.

\subsection{Using Probing to Investigate UX in the Factory Context}

To investigate HCI issues within the factory context, some studies have been conducted using different methods. Reul [16] reports on the improvement of usability of control software for industrial test systems. By using a contextual inquiry he could better understand the users' tasks and identify wishes of different user groups. For the redesign of the control room of a large factory Sagar [18] used contextual inquiry and observation as methods to gather information and understand the different tasks of the operators. A state of the art on control room design for industrial plants (e.g., power plants) has been published by the Technical Research Institute of Sweden [4]. Other reports cover usability issues in complex industrial control systems [14] especially focusing on nuclear power plants. There is no research as far as we are aware of, using more creative and engaging methods, such as probing, in the factory context.

The probing approach is used to help designers understand and specify the context of use and to support them to produce design solutions [12]. Probes/probe packages including creative materials, such as diaries, cameras, postcards, etc., are in general used as a means to offer inspiration for the designers or as a means to gather qualitative user information [2]. Originally developed by Gaver et al. [5], cultural probe packages contained open-ended questions, provocative and oblique tasks to support early participant engagement within a design process. Probes record a point of view, in the moment experience by making particular action, places, objects, people, etc. visible as well as motivations, wishes, desires, emotions, and intentions [6] [9].

The central element of probing is that users are given materials to describe their life or their work in a self-motivated way within the respective context. Users thus reveal new insights into practices and thoughts that cannot be revealed by classical approaches like questionnaires, interviews and observation. Probing offers an unobtrusive way to investigate people's attitudes and goes beyond reflective opinion gathering, as it fosters creativity. As Lucero et al. [11] point out, probes can - among other goals - facilitate looking into participants' daily practices and finding inspiration for new concepts.

Conducting UX research in such a particular context as the factory, using a design inspired method, was considered relevant for collecting feedback from the workers and providing valuable insights on further improvements of the work processes. 


\section{Field Study Set Up}

The probing method described in the previous section had to be adapted for studying the operators' experiences in the factory context due to the nature of the study environment and the limitations of the clean room. In the following we will give an overview of the target objectives and present the detailed study set up.

\subsection{Goals and Objectives}

The following objectives were defined for the conducted study (combining goals for UX research for this particular context and methodological insights):

(1) Investigate user experience of workers within the factory context.

(2) Apply a creative approach, inspired by probing, which is applicable for this context in order to investigate workers' experiences.

The first objective aimed at understanding UX within the factory environment, mainly the clean room. Thereby, we also tried to find out more about factors influencing UX, including aspects like the social network and interactions of the operators. By means of a creative stimuli approach, the study participants were asked to express themselves and explain their viewpoints concerning work with different devices and user interfaces within the clean room (e.g., see [13]). The second objective deals with the issue of how to adapt the probing method in a factory context to get insights on UX. For example, the time constraints of workers heavily influenced the creation of the study set up and the probing materials. Apart from the fact, that operators don't have the time to fill in probe packages during their work processes and the time slots before and after their individual shifts are too short to get reasonable results, we had to modify our set up to fit the contextual circumstances. Especially, the clean room poses certain limitations to classic probing materials, e.g. no normal paper and pen or cameras as material are allowed.

\subsection{Study Set Up and Materials}

After considering different procedural possibilities with the management (like handing out material to the operators for usage at their home, providing probing sets in the cafeteria, etc.) we decided to use one of the regularly scheduled instruction sessions to distribute the probe packages. These sessions are held at fixed intervals (approx. once a month), usually one hour before the normal shift starts. They are used to teach operators new skills or instruct them about changes/updates of the systems they are working with. These training sessions seemed ideal for carrying out our probing study. Although limited in time, this set up had the advantages, that the workers are already in the work context and do not need to use their spare time to do the probing (which is critical in this context). The study has been conducted on $12^{\text {th }}$ of October 2010 at a one-hour instruction session at the manufacturing plant. Participants $(\mathrm{N}=55)^{1}$ were operators working at the clean room. Two researchers explained the tasks and the aims of the study to the participants. Furthermore they

\footnotetext{
${ }^{1}$ No socio-demographic data were collected as it was not seen as relevant for this initial study and in order to keep the anonymity of the operators as requested by the management.
} 
gave a short overview of the different probing sets available. The probing material consisted of a variety of different materials, each focusing on a specific topic or task. The probing material included eight probing sets and was divided into two categories.

(1) Probes focusing on UX of workers and working conditions influencing the UX This category comprised six probing sets. The first three probing sets aimed at investigating current factors that account for the UX of workers within the factory context. They included questions about current mistakes that occur regularly, namely disturbing issues (see Fig. 1, "Things that bother me") and asked the participants to express wishes to facilitate work (see Fig. 1, "The magic fairy").

\section{Things that bother me}

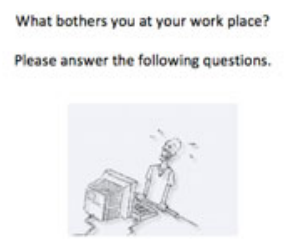

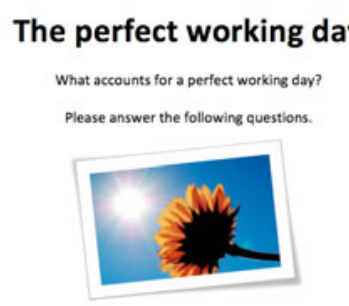

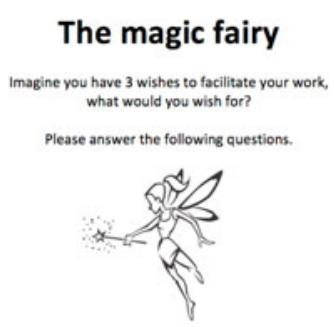

Fig. 1. Three examples of probes used in the factory context

The other three probing sets were designed to reveal more insights into daily work practices of the workers and factors that influence the UX. The first set wanted the workers to explain what constitutes a perfect workday (see Fig. 1, "The perfect working day"). The second set asked workers to describe their "neighbourhood", thus investigating to what extend the workers collaborate with others and how they experience the collaboration. The third probing material asked workers to draw their "level of stress for a typical day" on a time-graph, and explain highs and lows.

\section{(2) Probes dealing with feedback for existing and planned interfaces}

Aiming at involving users into the design process of interfaces we developed two probing sets that asked for their ideas and opinions on interfaces. In the first set they had to rearrange an existing interface according to their preferences grounded in their work practises. The second set was similar, but focused an interface that was currently developed. As we focus on UX within this paper, we do not present these two probes. Each of these probing sets contained a separate, quick description of how to use the material, including pens, scissors and glue. Every participant was able to choose one or more sets of probing material, depending on his/her interests. In the following the findings on UX on the factory context are presented.

\section{Relevant Findings on UX}

The collected probes ( $\mathrm{N}=75$ in total, of which 32 were relevant for UX) were transcribed as well as scanned in order not to lose any not-textual information. They were analysed using NVivo, a software package to support qualitative research 
(http://www.qsrinternational.com). We conducted a qualitative content analysis supported by statistical measures such as word frequency counts and rankings. Based on this analysis of the probing materials, insights on the UX factors stress, usability/ergonomics, and emotion could be gained. Moreover, social aspects turned out as a major influencing factor on UX in the factory. These factors (stress, usability/ergonomics, emotion, social aspects) can be seen as important in the factory context, as they were mentioned by the workers themselves, without giving them any triggers (open questions of the probes "Perfect Working Day", „Things that bother me“, „Magic Fairy“). Furthermore, specific insights on stress were revealed by the probe "On Stress". Table 1 gives an overview on the probes revealing insights on specific UX and influencing factors.

Table 1. Factors revealed from different Probes

\begin{tabular}{lcccc}
\hline & Stress & $\begin{array}{c}\text { Usability/ } \\
\text { Ergonomics }\end{array}$ & Emotion & Social Aspects \\
\hline $\begin{array}{l}\text { "Perfect Working } \\
\begin{array}{l}\text { Day" }(\mathrm{N}=10) \\
\text {,Things that bother } \\
\text { me“ }(\mathrm{N}=9)\end{array}\end{array}$ & $\mathrm{x}$ & $\mathrm{x}$ & $\mathrm{x}$ & \\
\hline „Magic Fairy" $(\mathrm{N}=3)$ & $\mathrm{x}$ & $\mathrm{x}$ \\
\hline "On Stress" $(\mathrm{N}=10)$ & $\mathrm{x}$ & & $\mathrm{x}$ \\
\hline
\end{tabular}

Stress: Stress turned out to be of high relevance for the workers' experience in the factory context, as it was mentioned often in different probes. When asking the participants how a perfect working day is characterized, stress was mentioned most often (in $80 \%$ of the probes). In particular, a perfect working day is mostly characterized by the absence of negative characteristics like stress or fear. Reasons mostly mentioned for stress are the end of a shift and shift change. At this time, the remaining work must be organized according to the established informal rules to avoid interruptions in the working process and enable a smooth takeover by the next shift group. Most of the time during a shift is experienced as stressful $(67 \%$ on average).

Usability/Ergonomics: The workers perceive usability and ergonomics as important. This factor is closely connected to the factor stress, as usability problems are often mentioned as a source of stress. When asking workers what bothers them in their work, they often mention usability and work organization (21\% of mentions). For example, workers bother when the machines are difficult to handle, or they do not like when there is not enough space for acting. Vice versa, a perfect working day is characterized by the prevention of errors and (bad) equipment (i.e. usability) (24\% of mentions). Thus, the absence of usability problems is the second frequently mentioned reason for a perfect working day surpassed only by the absence of stress. 
Emotion: At first glance, emotion does not seem to play important role in factory context. Due to the rather open design of some cards, we were able to identify emotion as a relevant aspect of UX in the factory. When analysing the probes in detail, it turned out that negative emotions like fear and anger are mentioned in relation to work. Positive emotions cannot be found in the probes. As most of our questions focused on drawbacks and deficiencies at work, the occurrence of negative emotions can be partially explained by the design of the probes.

Social Aspects: Social aspects probably represent the most important influencing factor on UX in the factory context. Probes concerning the social aspect show that workers desire more positive feedback from the boss or superiors, appreciate the reliability of colleagues, and demand equal treatment for all operators. When participants have three wishes, they mostly mention social aspects to be improved (e.g. better cooperation between colleagues). Asking workers for things that bother them at work, social aspects are often mentioned (e.g., staff, equal treatment, social contact; mentioned in $48 \%$ of the probes).

\section{Discussion and Conclusions}

No doubt, user experience (UX) has become of high relevance within the HCI community. Especially when the focus of HCI shifted from the work context to the more private and home context, the UX concept appeared. Designing for a fulfilling and enjoyable interaction with interactive systems has become a main goal for interaction designers. However, when it comes back to the work context, what significance can or should UX have in this context? Our study results showed that UX factors, such as emotions, stress, and social aspects, play an important role in the daily working routine of operators in a semiconductor factory. Investigating the workers experiences in this particular context was one of the main goals of our presented study using creative probes as stimuli. The results showed that the absence of stress significantly contributes to a perfect working day and that the end of a shift is experienced as the most stressful part of the daily working routine, as this is the most critical point of time for a fluent working process.

We further aimed at developing a creative approach applicable for the factory context. Probes can provide a good input for UX in the factory context. Open and unstructured probing cards (e.g., "Make a wish", "Things that bother me") give hints on which UX or influencing factors are important for workers. Thus, first insights on relevant UX factors can be gained. In a second step, insights on specific factors could be extended by triggering questions on specific factors (e.g., "On Stress"). Finally, probing has proven its relevance for investigating UX in the factory context. The adapted probing method turned out to be an appropriate method for providing easy and fast feedback of workers taking into consideration the time constraints and contextual circumstances. Thus, we developed an increased understanding of individuals' as well as on the groups of operators' user experience. As a next step we plan to link UX research back to user interface design and specific interaction problems in order to improve workers' experience in their daily work routines. 
Acknowledgments. The financial support by the Federal Ministry of Economy, Family and Youth and the National Foundation for Research, Technology and Development is gratefully acknowledged (Christian Doppler Laboratory for "Contextual Interfaces". Special thanks go to Florian Pöhr for his effort in the study.

\section{References}

1. Alben, L.: Quality of experience: defining the criteria for effective interaction design. Interactions 3(3), 11-15 (1996)

2. Arhippainen, L., Tahti, M.: Empirical evaluation of user experience in two adaptive mobile application prototypes. In: Proc. of the 2nd International Conference on Mobile and Ubiquitous Multimedia, pp. 27-34 (2003)

3. Brown, J.A.C.: The social psychology of industry: Human relations in the factory. Penguin Books, Harmondsworth (1954)

4. Ericson, M., Strandén, L., Emardson, R., Pendrill, L.F.: prestudy - State of the art survey of control rooms. SP Technical Research Institute of Sweden (2008)

5. Gaver, B., Dunne, T., Pacenti, E.: Design: Cultural probes. Interactions 6(1), 21-29 (1999)

6. Graham, C., Rouncefield, M., Gibbs, M., Vetere, F., Cheverst, K.: How probes work. In: Proc. of the 19th Australasian Conference on Computer-Human Interaction, pp. 29-37. ACM, NY (2007)

7. Hassenzahl, M., Tractinsky, N.: User experience - a research agenda. Behaviour \& Information Technology 25(2), 91-97 (2006)

8. Hassenzahl, M., Platz, A., Burmester, M., Lehner, K.: Hedonic and ergonomic quality aspects determine a software's appeal. In: Proc. CHI 2000, pp. 201-208. ACM, NY (2000)

9. Hemmings, T., Crabtree, A., Rodden, T., Clarke, K., Rouncefield, M.: Probing the probes. In: Proc. of the Participatory Design Conference, pp. 23-25 (2002)

10. Law, E.L.C., van Schaik, P.: Editorial: Modelling user experience - an agenda for research and practice. Interacting with Computers 22(5), 313-322 (2010)

11. Lucero, A., Lashina, T., Diederiks, E., Mattelmaki, T.: How probes inform and influence the design process. In: Proc. DPPI 2007, pp. 377-391. ACM, NY (2007)

12. Mattelmaki, T.: Design probes. University of Art and Design Helsinki, PhD thesis (2006)

13. Meech, J.: Intelligent systems for operator support. IEE Colloquium on HCI: Issues for the Factory, 2/1-2/6 (1991)

14. Meschtscherjakov, A., Reitberger, W., Pöhr, F., Tscheligi, M.: The operator guide: An ambient persuasive interface in the factory. In: Proc. of the First International Joint Conference on Ambient Intelligence 2010. LNCS, pp. 117-126. Springer, Heidelberg (2010)

15. Norros, L., Savioja, P.: Usability Evaluation of Complex Systems - A literature review. STUK - Radiation and Nuclear Safety Authority, Finland (2004)

16. Reul, M.: Bringing usability to industrial control systems. In: Proc. of CHI EA 2009, pp. 3335-3340. ACM, NY (2009)

17. Robinson, M.A.: Work sampling: Methodological advances and new applications. Human Factors and Ergonomics in Manufacturing \& Service Industries 20, 42-60 (2010)

18. Sagar, M.: User interface prototyping as seen from three viewpoints in the context of a supervision system. In: Proc. of the 15eme Conference Francophone sur l'Interaction Homme-Machine, pp. 190-197. ACM, New York (2003) 